Article

\title{
Systems of Variational Inequalities with Nonlinear Operators
}

\author{
Lu-Chuan Ceng ${ }^{1}\left[\right.$ and Qing Yuan ${ }^{2, *}$ (1) \\ 1 Department of Mathematics, Shanghai Normal University, Shanghai 200234, China; zenglc@hotmail.com \\ 2 School of Mathematics and Statistics, Linyi University, Linyi 276000, China \\ * Correspondence: yuanqing@lyu.edu.cn
}

Received: 2 March 2019; Accepted: 3 April 2019; Published: 9 April 2019

check for updates

\begin{abstract}
In this work, we concern ourselves with the problem of solving a general system of variational inequalities whose solutions also solve a common fixed-point problem of a family of countably many nonlinear operators via a hybrid viscosity implicit iteration method in 2 uniformly smooth and uniformly convex Banach spaces. An application to common fixed-point problems of asymptotically nonexpansive and pseudocontractive mappings and variational inequality problems for strict pseudocontractive mappings is also given in Banach spaces.
\end{abstract}

Keywords: implicit iterations; variational inequalities; lipschitzian pseudocontractions; asymptotically nonexpansive mapping; fixed-point; banach space

\section{Introduction}

Let $E$ be a real Banach space whose topological dual space is denoted by $E^{*}$. Recall that the normalized duality mapping $J: E \rightarrow 2^{E^{*}}$ is defined by

$$
J(x)=\left\{\varphi \in E^{*}:\|\varphi\|=\|x\|,\langle x, \varphi\rangle=\|x\|^{2}\right\}, \quad \forall x \in E,
$$

where $\langle\cdot, \cdot\rangle$ is the duality pair on $E$ and $E^{*} . J$ is single-valued in a smooth Banach space. In the sequel, we shall denote by $j$ the single-valued duality mapping, that is, $j(x) \in J(x)$. Let $C$ be a convex closed set in $E$. A mapping $f: C \rightarrow C$ is said to be $\delta$-Lipschitzian on $C$ if $\delta \in(0,+\infty)$ and $\|f(x)-f(y)\| \leq$ $\delta\|x-y\|$ for all $x, y \in C$. If $\delta<1$, then $f$ is called a $\delta$-contraction mapping or a contraction mapping with coefficient $\delta$. Each contraction $f: C \rightarrow C$ has a unique fixed point from the well known the Banach contractive principal. A mapping $f: C \rightarrow C$ is said to be nonexpansive if it is Lipschitzian with $\delta=1$. We use $\operatorname{Fix}(f)$ to denote the set of fixed points of $f$, i.e., $\operatorname{Fix}(f)=\{x \in C: f(x)=x\}$. Moreover, a mapping $T: C \rightarrow C$ is said to be asymptotically nonexpansive [1] if there exists a sequence $\left\{\theta_{n}\right\} \subset[0,+\infty)$ with $\lim _{n \rightarrow \infty} \theta_{n}=0$ such that

$$
\left\|T^{n} x-T^{n} y\right\| \leq\|x-y\|+\theta_{n}\|x-y\|, \quad \forall x, y \in C, \forall n \geq 0 .
$$

If

$$
\limsup _{n \rightarrow \infty}\left(\sup _{x, y \in C}\left(\left\|T^{n} x-T^{n} y\right\|-\|x-y\|\right)\right) \leq 0,
$$

and $T$ enjoys the continuity, then $T$ is called an asymptotically nonexpansive mapping in the intermediate sense; see [2]. Throughout this paper, we assume

$$
c_{n}:=\max \left\{0, \sup _{x, y \in C}\left(\left\|T^{n} x-T^{n} y\right\|-\|x-y\|\right)\right\} .
$$


Hence, $c_{n} \geq 0 \forall n \geq 0, c_{n} \rightarrow 0(n \rightarrow \infty)$, and the definition is reduced to

$$
\left\|T^{n} x-T^{n} y\right\| \leq\|x-y\|+c_{n}, \quad \forall x, y \in C, \forall n \geq 0 .
$$

Recall that a mapping $T$ with domain $D(T)$ and range $R(T)$ in $E$ is called pseudocontractive if the inequality holds

$$
\|x-y\| \leq\|r((I-T) x-(I-T) y)+(x-y)\|, \quad \forall x, y \in D(T), \forall r>0 .
$$

From a result of Kato [3], we know that the notion of pseudocontraction is equivalent to the one that for each $x, y \in D(T)$, there exists $j(x-y) \in J(x-y)$ such that

$$
\langle T x-T y, j(x-y)\rangle \leq\|x-y\|^{2} .
$$

It is well known that the class of pseudocontractive mappings is a crucial generation of nonexpansive mappings. Moreover, focus on pseudocontractive mappings are also from their relation with the class of accretive mappings in Banach spaces (monotone in Hilbert spaces). A mapping $A$ with domain $D(A)$ and range $R(A)$ in $E$ is called accretive if for each $x, y \in D(T)$, there exists $j(x-y) \in J(x-y)$ such that

$$
\langle A x-A y, j(x-y)\rangle \geq 0 .
$$

It will be called a monotone mapping if the space is Hilbert. If for each $x, y \in D(A)$, there exists $j(x-y) \in J(x-y)$ such that

$$
\alpha\|A x-A y\|^{2} \leq\langle A x-A y, j(x-y)\rangle \text { for some } \alpha>0,
$$

then $A$ is called $\alpha$-inverse-strongly accretive.

Recently, fixed/zero points of pseudocontraction/accretive operators were investigated by many authors for solving various convex optimization problems; see [4-13] and the references therein.

Let $E$ be a smooth Banach space. Let $B_{1}, B_{2}$ be two non-self-mappings from $C$ to $E$. The general system of variational inequalities (GSVI) is to find $\left(x^{*}, y^{*}\right) \in C \times C$ such that

$$
\begin{cases}\left\langle\mu_{1} B_{1} y^{*}-y^{*}+x^{*}, j\left(x-x^{*}\right)\right\rangle \geq 0, & \forall x \in C, \\ \left\langle\mu_{2} B_{2} x^{*}-x^{*}+y^{*}, j\left(x-y^{*}\right)\right\rangle \geq 0, & \forall x \in C,\end{cases}
$$

where $\mu_{1}$ and $\mu_{2}$ are two positive coefficients.

In particular, if $B_{1}=B_{2}=B$, then problem (3) reduces to the following system of variational inequalities (SVI) in Banach spaces:

Find $\left(x^{*}, y^{*}\right) \in C \times C$ such that

$$
\begin{cases}\left\langle\mu_{1} B y^{*}-y^{*}+x^{*}, j\left(x-x^{*}\right)\right\rangle \geq 0, & \forall x \in C, \\ \left\langle\mu_{2} B x^{*}-x^{*}+y^{*}, j\left(x-y^{*}\right)\right\rangle \geq 0, & \forall x \in C .\end{cases}
$$

Furthermore, if $x^{*}=y^{*}$, then we obtain the following variational inequality (VI) in Banach spaces: Find $x^{*} \in C$ such that

$$
\left\langle\mu B x^{*}, j\left(x-x^{*}\right)\right\rangle \geq 0, \quad \forall x \in C .
$$

Let $\operatorname{VI}(C, B)$ denote the set of solutions to problem (5). Whenever $E=H$ a real Hilbert space, it is easy to see that the GSVI (3) reduces to the following problem of finding $\left(x^{*}, y^{*}\right) \in C \times C$ such that

$$
\begin{cases}\left\langle\mu_{1} B_{1} y^{*}-y^{*}+x^{*}, x-x^{*}\right\rangle \geq 0, & \forall x \in C, \\ \left\langle\mu_{2} B_{2} x^{*}-x^{*}+y^{*}, x-y^{*}\right\rangle \geq 0, & \forall x \in C,\end{cases}
$$


which is called a GSVI in Hilbert spaces. In [11], the GSVI (6) was transformed into a fixed-point problem by Ceng, Wang and Yao in the following way.

Lemma 1. [14] For chosen $x^{*}, y^{*} \in C, x^{*}-y^{*}$ is a solution of GSVI (1.6) if and only if $x^{*} \in \operatorname{GSVI}\left(C, B_{1}, B_{2}\right)$, where $\operatorname{GSVI}\left(C, B_{1}, B_{2}\right)$ is the fixed-point set of the mapping $G:=P_{C}\left(P_{C}\left(I-\eta B_{2}\right)-\rho B_{1} P_{C}\left(I-\eta B_{2}\right)\right)$, and $y^{*}=P_{C}\left(I-\eta B_{2}\right) x^{*}$.

Recently, many authors studied problems (3)-(6) via projection-based methods in Hilbert or Banach spaces; see [15-22] and the references therein. In this paper, we introduce a hybrid viscosity implicit iteration method that is based on Korpelevich's extragradient method, the viscosity approximation method and the Mann iteration method for finding a common solution of the GSVI (3) for two inverse-strongly accretive mappings, a common fixed-point problem (CFPP) of a countable family of uniformly Lipschitzian pseudocontractive mappings and an asymptotically nonexpansive mapping in the intermediate sense. We prove the strong convergence of the proposed method to a common solution of the GSVI (3) and the CFPP, which solves a certain variational inequality on their common solution set in 2-uniformly smooth and uniformly convex Banach spaces. Additionally, we give an application to solve CFPPs of asymptotically nonexpansive and pseudocontractive mappings, and variational inequality problems for strict pseudocontractive mappings in Banach spaces.

\section{Preliminaries}

Throughout this paper we write $x_{n} \rightarrow x$ (respectively, $x_{n} \rightarrow x$ ) to indicate that the sequence $\left\{x_{n}\right\}$ converges weakly (respectively, strongly) to $x$. Without loss of generality, we assume that $E$ is a real Banach space and the dual will be presented by $E^{*}$ in this paper.

Definition 1. Let $\left\{S_{n}\right\}_{n=0}^{\infty}$ be a vector sequence of pseudocontractive continuous self-mappings on $C$, a convex closed convex subset of Banach space E. Recall that $\left\{S_{n}\right\}_{n=0}^{\infty}$ is said to be a countable family of $\ell$-uniformly Lipschitzian pseudocontractive self-mappings provided that there exists a constant $\ell>0$ such that each $S_{n}$ is a

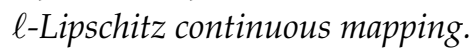

In a smooth Banach space $E$, an operator $A$ is said to be strongly positive if there exists a constant $\bar{\gamma}>0$ with the property

$$
\|a I-b A\|=\sup _{\|x\| \leq 1}|\langle(a I-b A) x, j(x)\rangle|,\langle A x, j(x)\rangle \geq \bar{\gamma}\|x\|^{2} a \in[0,1], b \in[-1,1],
$$

where $I$ is the identity mapping and $j(\cdot)$ is the single-valued normalized duality mapping.

Recall that a Banach space $E$ is said to be strictly convex if for any $x, y \in\{x \in E:\|x\|=1\}$, $x \neq y \Rightarrow\left\|\frac{x+y}{2}\right\|<1$. It is also said to be uniformly convex if for each $\epsilon \in(0,2]$, there exists $\delta>0$ such that for any $x, y \in\{x \in E:\|x\|=1\},\|x-y\| \geq \epsilon \Rightarrow\|x+y\| \leq 2-2 \delta$. Clearly, if $E$ is uniformly convex, then it is strictly convex. A Banach space $E$ is said to have a Gâteaux differentiable norm if the limit

$$
\lim _{t \rightarrow 0} \frac{\|x+t y\|-\|x\|}{t}
$$

exists for each $x, y \in\{x \in E:\|x\|=1\}$ and in this case we call $E$ smooth; $E$ is said to have a uniformly Gâteaux differentiable norm if for each $y \in\{x \in E:\|x\|=1\}$, the above limit is attained uniformly for $x \in\{x \in E:\|x\|=1\}$. Moreover, it is said to have a uniformly Fréchet differentiable norm if the above limit is attained uniformly for $x, y \in\{x \in E:\|x\|=1\}$ and in this case we call $E$ uniformly smooth. The norm of $E$ is said to be the Fréchet differentiable if for each $x \in\{x \in E:\|x\|=1\}$, the 
above limit is attained uniformly for $y \in\{x \in E:\|x\|=1\}$. The modulus of smoothness of $E$ is defined by

$$
\varrho(\tau)=\sup \left\{\frac{(\|x+y\|+\|x-y\|)-2}{2}: x, y \in E,\|x\|=1,\|y\|=\tau\right\},
$$

where $\varrho:[0, \infty) \rightarrow[0, \infty)$ is a function. It is known that $E$ is uniformly smooth if and only if $\lim _{\tau \rightarrow 0} \frac{\varrho(\tau)}{\tau}=0$. Let $q$ be a fixed real number with $1<q \leq 2$. A Banach space $E$ is said to be $q$-uniformly smooth if there exists a constant $\kappa>0$ such that $\frac{\varrho(\tau)}{\kappa} \leq \tau^{q}$ for all $\tau>0$. From [23], we know the following relation. Let $q$ be a fixed number with $1<q \leq 2$ and $E$ be a Banach space. Then $E$ is $q$-uniformly smooth if and only if there exists a constant $c>0$ such that

$$
\|x\|^{q}+\|\kappa y\|^{q} \geq \frac{\|x+y\|^{q}+\|x-y\|^{q}}{2}, \quad \forall x, y \in E .
$$

The best constant $\kappa$ in the above inequality is called the $q$-uniformly smooth constant of $E$; see [23] for more details. In addition, no Banach space is $q$-uniformly smooth for $q>2$; see [24] for more details. If $E$ be a 2-uniformly smooth Banach space. Then

$$
\|x+y\|^{2}-\|x\|^{2} \leq 2\langle y, j(x)\rangle+2\|\kappa y\|^{2}, \quad \forall x, y \in E,
$$

where $\kappa$ is the 2-uniformly smooth constant of $E$.

In particular, if $E$ is a Hilbert space, then the duality pairing $\langle\cdot, \cdot\rangle$ reduces to the inner product, $j=I$ the identity mapping of $E$, and $\kappa=\sqrt{2} / 2$.

For $q>1$, the generalized duality mapping $J_{q}: E \rightarrow 2^{E^{*}}$ is defined by

$$
J_{q}(x)=\left\{\varphi \in E^{*}:\|\varphi\|=\|x\|^{q-1},\langle x, \varphi\rangle=\|x\|^{q}\right\}, \quad \forall x \in E .
$$

In particular, $J=J_{2}$ is called the normalized duality mapping. It is known that $J(x)=\frac{J_{q}(x)}{\|x\|^{q-2}}$ for all $x \in E$. If $E$ is a Hilbert space, then $J=I$ (the identity mapping). Recall that the following statements hold:

(1) if $E$ is smooth, then $J$ is norm-to-weak* continuous single-valued on $E$;

(2) if $E$ is uniformly smooth, then $J$ is norm-to-norm uniformly continuous single-valued on bounded subsets of $E$;

(3) if $E$ has a uniformly Gáteaux differentiable norm, then $J$ is norm-to-weak* uniformly continuous single-valued on bounded subsets of $E$;

Proposition 1. (see [25]). Let $C$ be a convex nonempty closed set in a Banach space E. Let $S_{0}, S_{1}, \ldots$ be a sequence of mappings of $C$ into itself. Suppose $\sum_{n=1}^{\infty} \sup \left\{\left\|S_{n} x-S_{n-1} x\right\|: x \in C\right\}<\infty$. For each $y \in C$, $\left\{S_{n} y\right\}$ converges in norm to some point of $C$. Moreover, let $S$ be a mapping defined by $S y=\lim _{n \rightarrow \infty} S_{n} y$ for all $y \in C .\left\{\left\|S x-S_{n} x\right\|: x \in C\right\} \rightarrow 0$ as $n \rightarrow \infty$.

Proposition 2. (see [26]). Let $C$ be a convex closed set in a Banach space $E$ and $T: C \rightarrow C$ be a strong continuous pseudocontraction mapping. Then, $T$ has a fixed point. Indeed, it is the unique fixed point in $C$ for $T$.

Let $D$ be a nonempty set in $C$ and let $\Pi$ be a mapping from $C$ to $D$. Then $\Pi$ is said to be a sunny if $\Pi[(1-t) \Pi(x)+t x]=\Pi(x)$, when $(1-t) \Pi(x)+t x \in C$ for all $x \in C$ and $t \geq 0$. A mapping $\Pi$ of $C$ into itself is called a retraction if $\Pi^{2}=\Pi$. If a mapping $\Pi$ of $C$ into itself is a retraction, then $\Pi(z)=z$ for each $z \in R(\Pi)$, where $R(\Pi)$ is the range of $\Pi$. A subset $D$ of $C$ is called a sunny nonexpansive retract of $C$ if there exists a sunny nonexpansive retraction from $C$ onto $D$.

In a smooth Banach space $E$, a duality mapping $J$ is said to be weakly sequentially continuous [27], if for each $\left\{x_{n}\right\} \subset E$ with $x_{n} \rightarrow x$, then $\left\{j\left(x_{n}\right)\right\}$ converges weakly* to $j(x)$. In [27], Gossez and Lami 
Dozo showed that a space with a weakly continuous duality mapping satisfies Opial's condition. Conversely, we know from [28] that if a space satisfies Opial's condition and has a uniformly Gáteaux differentiable norm, then it has a weakly continuous duality mapping.

Proposition 3. (see [29]). Let $C$ be a nonempty closed convex subset of a smooth Banach space E, $D$ be a nonempty subset of $C$ and $\Pi$ be a retraction of $C$ onto $D$. Then the following are equivalent:

(i) $\Pi$ is sunny and nonexpansive;

(ii) $\langle x-y, j(\Pi(x)-\Pi(y))\rangle \geq\|\Pi(x)-\Pi(y)\|^{2}, \forall x, y \in C$;

(iii) $\langle x-\Pi(x), j(y-\Pi(x))\rangle \leq 0, \forall x \in C, y \in D$.

If $E$ is a Hilbert space, then a sunny nonexpansive retraction $\Pi_{C}$ of $E$ onto $C$ coincides with the nearest projection of $E$ onto $C$ and it is well known that if $C$ is a convex closed set in a reflexive Banach space $E$ with a uniformly Gáteaux differentiable norm and $D$ is a nonexpansive retract of $C$, then it is a sunny nonexpansive retract of $C$; see, e.g., $[30,31]$ and the references therein.

To prove our main results, we need to use some lemmas in the sequel. The following Lemma is an immediate consequence of the subdifferential inequality of the function $\frac{1}{2}\|\cdot\|^{2}$.

Lemma 2. [32] Let E be a real Banach space and $J$ be the normalized duality mapping on E. Then for any given $x, y \in E$, the following inequality holds:

$$
\|x+y\|^{2}-\|x\|^{2} \leq 2\langle y, j(x+y)\rangle, \quad \forall j(x+y) \in J(x+y) .
$$

If $C$ is a convex closed set in a smooth Banach space $E$ and $\Pi_{C}$ a sunny nonexpansive retraction from $E$ onto $C$, we have

$$
\operatorname{VI}(C, B)=\operatorname{Fix}\left(\Pi_{C}(I-\lambda B)\right) .
$$

where $B: C \rightarrow E$ be an accretive mapping and $\lambda>0$,

Using Proposition 3, we immediately obtain the following lemmas.

Lemma 3. Let $C$ be a nonempty closed convex subset of a smooth Banach space $E$ and $B_{1}, B_{2}: C \rightarrow E$ be two nonlinear mappings. Let $\Pi_{C}$ be a sunny nonexpansive retraction from $E$ onto $C$. For given $x^{*}, y^{*} \in C,\left(x^{*}, y^{*}\right)$ is a solution of the GSVI (3) if and only if $x^{*} \in \operatorname{GSVI}\left(C, B_{1}, B_{2}\right)$ where $\operatorname{GSVI}\left(C, B_{1}, B_{2}\right)$ is the set of fixed points of the mapping

$$
G:=\Pi_{C}\left(\Pi_{C}\left(I-\mu_{2} B_{2}\right)-\mu_{1} B_{1} \Pi_{C}\left(I-\mu_{2} B_{2}\right)\right)
$$

and $y^{*}=\Pi_{C}\left(I-\mu_{2} B_{2}\right) x^{*}$.

Lemma 4. Let $C$ be a nonempty closed convex subset of a 2-uniformly smooth Banach space E. Let the mapping $A: C \rightarrow E$ be $\alpha$-inverse-strongly accretive. Then, for any given $\lambda \geq 0$,

$$
\|(I-\lambda A) x-(I-\lambda A) y\|^{2}-\|x-y\|^{2} \leq 2 \lambda\left(\kappa^{2} \lambda-\alpha\right)\|A x-A y\|^{2} .
$$

In particular, if $0 \leq \lambda \kappa^{2} \leq \alpha$, then $I-\lambda A$ is a nonexpansive operator. Let $\Pi_{C}$ be a sunny nonexpansive retraction from $E$ onto $C$. Let the mappings $B_{1}, B_{2}: C \rightarrow E$ be $\alpha$-inverse-strongly accretive and $\beta$-inverse-strongly accretive, respectively. Let the mapping $G: C \rightarrow C$ be defined as $G:=\Pi_{C}\left(I-\mu_{1} B_{1}\right) \Pi_{C}\left(I-\mu_{2} B_{2}\right)$. If $0 \leq \mu_{1} \kappa^{2} \leq \alpha$ and $0 \leq \mu_{2} \kappa^{2} \leq \beta$, then $G: C \rightarrow C$ is nonexpansive.

Let $C$ be a nonempty closed convex subset of a uniformly convex Banach space $E$ and $T: C \rightarrow C$ be an asymptotically nonexpansive mapping in the intermediate sense. Given any bounded subset 
$\mathcal{K} \subset C$. For every $\varepsilon>0$ and every integer $n \geq 2$ there exist an integer $N_{\varepsilon} \geq 1$ and $\delta_{\varepsilon}>0$, where both $N_{\varepsilon}$ and $\delta_{\varepsilon}$ are independent of $n$, such that if $k \geq N_{\varepsilon}, z_{1}, z_{2}, \ldots, z_{n} \in \mathcal{K}$ and if

$$
\left\|z_{i}-z_{j}\right\|-\left\|T^{k} z_{i}-T^{k} z_{j}\right\| \leq \delta_{\varepsilon}
$$

for $1 \leq i, j \leq n$, then

$$
\left\|T^{k}\left(\sum_{i=1}^{n} \lambda_{i} z_{i}\right)-\sum_{i=1}^{n} \lambda_{i} T^{k} z_{i}\right\|<\varepsilon
$$

for all $\lambda=\left(\lambda_{1}, \lambda_{2}, \ldots, \lambda_{n}\right)$ such that $\lambda_{i} \geq 0$ for $i=1,2, \ldots, n$ and $\sum_{i=1}^{n} \lambda_{i}=1$; see ([33], Lemma 4 ) for details.

From the above results, we know that if $\left\{x_{m}\right\}_{m=0}^{\infty}$ is a sequence in $C$ converging weakly to $x$ and if $\lim _{m \rightarrow \infty}\left\|x_{m}-T x_{m}\right\|=0$, then $T x=x$, where $T: C \rightarrow C$ is a uniformly continuous self-mapping on $C$, which is asymptotically nonexpansive in the intermediate sense.

Lemma 5. (see [34]). Let E be a smooth and uniformly convex Banach space, and let $r>0$. Then there exists a strictly increasing, continuous, and convex function $g:[0,2 r] \rightarrow \mathbf{R}, g(0)=0$ such that

$$
g(\|x-y\|)+2\langle x, j(y)\rangle \leq\|x\|^{2}+\|y\|^{2}, \quad \forall x, y \in\{x \in E:\|x\| \leq r\} .
$$

Lemma 6. (see [35]). Let $E$ be a reflexive Banach space, $C$ be a convex nonempty, closed subset of $E$, and $T: C \rightarrow E$ be a nonexpansive mapping. Suppose that E admits a weakly sequentially continuous duality mapping. Then the mapping $I-T$ is demiclosed on $C$, where I is the identity mapping.

Lemma 7. (see [36]). Let $\left\{a_{n}\right\}$ be a sequence of nonnegative real numbers satisfying

$$
a_{n+1} \leq a_{n}+s_{n} t_{n}+v_{n}-s_{n} a_{n}, \quad \forall n \geq 0,
$$

where $\left\{s_{n}\right\},\left\{t_{n}\right\}$ and $\left\{v_{n}\right\}$ satisfy the conditions:

(i) $\limsup _{n \rightarrow \infty} t_{n} \leq 0$;

(ii) $\left\{s_{n}\right\} \subset[0,1]$ and $\sum_{n=0}^{\infty} s_{n}=\infty$;

(iii) $v_{n} \geq 0, \forall n \geq 0$, and $\sum_{n=0}^{\infty} v_{n}<\infty$.

Then $\lim _{n \rightarrow \infty} a_{n}=0$.

\section{Main Results}

In this section, we suggest and analyze a hybrid viscosity implicit iteration method for solving the GSVI (3) with the hierarchical variational inequality (HVI) constraint for countably many uniformly Lipschitzian pseudocontractive mappings and an asymptotically nonexpansive mapping in the intermediate sense in a 2-uniformly smooth and uniformly convex Banach space.

Theorem 1. Let $C$ be a convex closed set in a 2-uniformly smooth and uniformly convex Banach space $E$ which admits a weakly sequentially continuous duality mapping. Let $\Pi_{C}$ be a sunny nonexpansive retraction from $E$ onto $C$. Let the mappings $B_{1}, B_{2}: C \rightarrow E$ be $\alpha$-inverse-strongly accretive and $\beta$-inverse-strongly accretive, respectively. Let $f: C \rightarrow C$ be a contraction mapping with coefficient $\gamma \in[0,1)$ and $F: E \rightarrow E$ be a strongly positive linear bounded operator with the coefficient $\bar{\gamma}$ such that $0<\gamma<\bar{\gamma} \theta$ and $0<\theta \leq\|F\|^{-1}$. Let $T: C \rightarrow C$ be uniformly continuous and asymptotically nonexpansive mapping in the intermediate sense, and $\left\{S_{n}\right\}_{n=0}^{\infty}$ be a countable family of $\ell$-uniformly Lipschitzian pseudocontractive self-mappings on $C$ such that $\Omega:=\bigcap_{n=0}^{\infty} \operatorname{Fix}\left(S_{n}\right) \cap \operatorname{GSVI}\left(C, B_{1}, B_{2}\right) \cap \operatorname{Fix}(T) \neq \varnothing$ where $\operatorname{GSVI}\left(C, B_{1}, B_{2}\right)$ is the fixed-point set of the mapping $G:=\Pi_{C}\left(\Pi_{C}\left(I-\mu_{2} B_{2}\right)-\mu_{1} B_{1} \Pi_{C}\left(I-\mu_{2} B_{2}\right)\right)$ with $0<\mu_{1} \kappa^{2}<\alpha$ and $0<\mu_{2} \kappa^{2}<\beta$ for $\kappa$ the 
2-uniformly smooth constant of E. Assume that $\sum_{n=0}^{\infty} c_{n}<\infty$, where $c_{n}$ is defined by (2). For arbitrarily given $x_{0} \in C$, let $\left\{x_{n}\right\}$ be the sequence generated by

$$
\left\{\begin{array}{l}
u_{n}=\beta_{n}\left(x_{n}-S_{n} u_{n}\right)+S_{n} u_{n} \\
z_{n}=\Pi_{C}\left(u_{n}-\mu_{2} B_{2} u_{n}\right), \\
y_{n}=\Pi_{C}\left(z_{n}-\mu_{1} B_{1} z_{n}\right), \\
x_{n+1}=\Pi_{C}\left[\left(T^{n} y_{n}-\alpha_{n} \theta F T^{n} y_{n}\right)+\alpha_{n} f\left(x_{n}\right)\right], \quad \forall n \geq 0
\end{array}\right.
$$

where $\left\{\alpha_{n}\right\}$ and $\left\{\beta_{n}\right\}$ are the sequences in $[0,1]$ satisfying the following conditions:

(i) $\lim _{n \rightarrow \infty} \alpha_{n}=0, \sum_{n=1}^{\infty}\left|\alpha_{n}-\alpha_{n-1}\right|<\infty$ and $\sum_{n=0}^{\infty} \alpha_{n}=\infty$,

(ii) $0<\liminf _{n \rightarrow \infty} \beta_{n} \leq \limsup _{n \rightarrow \infty} \beta_{n}<1$ and $\sum_{n=1}^{\infty}\left|\beta_{n}-\beta_{n-1}\right|<\infty$.

Assume that $\sum_{n=1}^{\infty} \sup _{x \in D}\left\|S_{n} x-S_{n-1} x\right\|<\infty$ for any bounded subset $D$ of $C$, and let $S$ be a mapping of $C$ into itself defined by $S x=\lim _{n \rightarrow \infty} S_{n} x$ for all $x \in C$, and suppose that $\operatorname{Fix}(S)=\bigcap_{n=0}^{\infty} \operatorname{Fix}\left(S_{n}\right)$. If $\sum_{n=0}^{\infty}\left\|T^{n+1} y_{n}-T^{n} y_{n}\right\|<\infty$, then $\left\{x_{n}\right\}$ converges strongly to $x^{*} \in \Omega$. In this case,

(a) $x^{*} \in \Omega$ solves the VI: $\left\langle f\left(x^{*}\right)-\theta F\left(x^{*}\right), j\left(x-x^{*}\right)\right\rangle \leq 0, \forall x \in \Omega$;

(b) $\left(x^{*}, y^{*}\right)$ is a solution of GSVI (1.3) with $y^{*}=\Pi_{C}\left(I-\mu_{2} B_{2}\right) x^{*}$.

Proof. First of all, from $\alpha_{n} \rightarrow 0(n \rightarrow \infty)$, we may assume, without loss of generality, that $\alpha_{n} \leq \frac{\|F\|^{-1}}{\theta}$ for all $n \geq 0$. Since $F$ is strongly positive linear bounded, it follows that $1-\alpha_{n} \bar{\gamma} \theta \geq\left\|I-\alpha_{n} \theta F\right\|$. Taking into account that $\left\{\beta_{n}\right\}$ is bounded away from 0 and 1 , we may assume that $\left\{\beta_{n}\right\} \subset[a, b] \subset(0,1)$ for some $a, b \in(0,1)$. Please note that the mapping $G: C \rightarrow C$ is defined as $G:=\Pi_{C}\left(\Pi_{C}\left(I-\mu_{2} B_{2}\right)-\right.$ $\left.\mu_{1} B_{1} \Pi_{C}\left(I-\mu_{2} B_{2}\right)\right)$, where $0<\mu_{1} \kappa^{2} \leq \alpha$ and $0<\mu_{2} \kappa^{2} \leq \beta$ for $\kappa$ the 2-uniformly smooth constant of $E$. Therefore, by Lemma 3, we obtain that $G$ is nonexpansive. It is easy to see that for each $n \geq 0$ there exists a unique element $u_{n} \in C$ such that

$$
u_{n}=\beta_{n}\left(x_{n}-S_{n} u_{n}\right)+S_{n} u_{n}
$$

As a matter of fact, consider the mapping

$$
F_{n} x=\beta_{n}\left(x_{n}-S_{n} x\right)+S_{n} x, \quad \forall x \in C .
$$

Since $S_{n}: C \rightarrow C$ is a continuous pseudocontraction mapping, we deduce that

$$
\left(1-\beta_{n}\right)\|x-y\|^{2} \geq\left(1-\beta_{n}\right)\left\langle S_{n} x-S_{n} y, j(x-y)\right\rangle=\left\langle F_{n} x-F_{n} y, j(x-y)\right\rangle, \quad \forall x, y \in C .
$$

Also, from $\left\{\beta_{n}\right\} \subset[a, b] \subset(0,1)$ we get $0<1-\beta_{n}<1$ for all $n \geq 0$. Thus, $F_{n}$ is a continuous and strong pseudocontraction mapping of $C$ into itself. By Proposition 2, we know that for each $n \geq 0$ there exists a unique element $u_{n} \in C$, satisfying (8). Therefore, it can be readily seen that the hybrid viscosity implicit iterative scheme (7) can be rewritten as

$$
\left\{\begin{array}{l}
u_{n}=\beta_{n}\left(x_{n}-S_{n} u_{n}\right)+S_{n} u_{n}, \\
y_{n}=G u_{n}, x_{n+1}=\Pi_{C}\left[\left(T^{n} y_{n}-\alpha_{n} \theta F T^{n} y_{n}\right)+\alpha_{n} f\left(x_{n}\right)\right], \quad \forall n \geq 0,
\end{array}\right.
$$

Next, we divide the rest of the proof into several steps.

Step 1. We claim that $\left\{x_{n}\right\},\left\{y_{n}\right\},\left\{z_{n}\right\},\left\{u_{n}\right\},\left\{f\left(x_{n}\right)\right\},\left\{T^{n} y_{n}\right\}$ and $\left\{F\left(T^{n} y_{n}\right)\right\}$ are bounded vector sequences. Indeed, take an element $p \in \Omega=\bigcap_{n=0}^{\infty} \operatorname{Fix}\left(S_{n}\right) \cap \operatorname{GSVI}\left(C, B_{1}, B_{2}\right) \cap \operatorname{Fix}(T)$ arbitrarily. Then 
we have $S_{n} p=p, G p=p$ and $T p=p$. Since each $S_{n}: C \rightarrow C$ is a pseudocontraction mapping, it follows that

$$
\begin{aligned}
\left\|p-u_{n}\right\|^{2} & =\left\langle\beta_{n}\left(p-x_{n}\right)+\left(1-\beta_{n}\right)\left(p-S_{n} u_{n}\right), j\left(p-u_{n}\right)\right\rangle \\
& =\left(1-\beta_{n}\right)\left\langle p-S_{n} u_{n}, j\left(p-u_{n}\right)\right\rangle+\beta_{n}\left\langle p-x_{n}, j\left(p-u_{n}\right)\right\rangle \\
& \leq\left(1-\beta_{n}\right)\left\|p-u_{n}\right\|^{2}+\beta_{n}\left\|x_{n}-p\right\|\left\|p-u_{n}\right\|,
\end{aligned}
$$

which hence yields

$$
\left\|p-u_{n}\right\| \leq\left\|p-x_{n}\right\|, \quad \forall n \geq 0
$$

Then we observe

$$
\left\|y_{n}-p\right\|=\left\|G u_{n}-p\right\| \leq\left\|u_{n}-p\right\| \leq\left\|x_{n}-p\right\| .
$$

Combining (9) and (11), we have

$$
\begin{aligned}
\left\|x_{n+1}-p\right\| & \leq\left\|\alpha_{n}\left(f\left(x_{n}\right)-\theta F(p)\right)+\left(I-\alpha_{n} \theta F\right) T^{n} y_{n}-\left(I-\alpha_{n} \theta F\right) p\right\| \\
& \leq \alpha_{n} \gamma\left\|x_{n}-p\right\|+\alpha_{n}\|f(p)-\theta F(p)\|+\left(1-\alpha_{n} \bar{\gamma} \theta\right)\left\|T^{n} y_{n}-p\right\| \\
& \leq \alpha_{n} \gamma\left\|x_{n}-p\right\|+\alpha_{n}\|f(p)-\theta F(p)\|+\left(1-\alpha_{n} \bar{\gamma} \theta\right)\left(\left\|y_{n}-p\right\|+c_{n}\right) \\
& \leq c_{n}+\alpha_{n}\|f(p)-\theta F(p)\|+\alpha_{n} \gamma\left\|x_{n}-p\right\|+\left(1-\alpha_{n} \bar{\gamma} \theta\right)\left\|x_{n}-p\right\| \\
& \leq c_{n}+\max \left\{\left\|x_{n}-p\right\|, \frac{\|f(p)-\theta F(p)\|}{\bar{\gamma} \theta-\gamma}\right\} .
\end{aligned}
$$

By induction, we get

$$
\left\|x_{n}-p\right\| \leq \sum_{n=0}^{\infty} c_{n}+\max \left\{\left\|x_{0}-p\right\|, \frac{\|f(p)-\theta F(p)\|}{\bar{\gamma} \theta-\gamma}\right\}, \quad \forall n \geq 0 .
$$

It immediately follows that $\left\{x_{n}\right\}$ is bounded, and so are the sequences $\left\{y_{n}\right\},\left\{u_{n}\right\},\left\{f\left(x_{n}\right)\right\},\left\{T^{n} y_{n}\right\}$ and $\left\{F\left(T^{n} y_{n}\right)\right\}$ (due to (10), (11) and the Lipschitz continuity of $f$ ). Taking into account that $\left\{S_{n}\right\}$ is $\ell$-uniformly Lipschitzian on $C$, we know that

$$
\ell\left\|u_{n}-p\right\|+\|p\| \geq \mid S_{n} u_{n}-p\|+\| p\|\geq\| S_{n} u_{n} \|,
$$

which implies that $\left\{S_{n} u_{n}\right\}$ is bounded. In addition, from Lemma 3 and $p \in \Omega \subset \operatorname{GSVI}\left(C, B_{1}, B_{2}\right)$, it also follows that $(p, q)$ is a solution of GSVI (3) where $q=\Pi_{C}\left(p-\mu_{2} B_{2} p\right)$. Please note that $z_{n}=\Pi_{C}\left(I-\mu_{2} B_{2}\right) u_{n}$ for all $n \geq 0$. Then by Lemma 4 we get

$$
\begin{aligned}
\left\|z_{n}\right\| & \leq\left\|\Pi_{C}\left(u_{n}-\mu_{2} B_{2} u_{n}\right)-\Pi_{C}\left(p-\mu_{2} B_{2} p\right)\right\|+\|q\| \\
& \leq\left\|\left(u_{n}-\mu_{2} B_{2} u_{n}\right)-\left(p-\mu_{2} B_{2} p\right)\right\|+\|q\| \\
& \leq\|q\|+\left\|u_{n}-p\right\| .
\end{aligned}
$$

This shows that $\left\{z_{n}\right\}$ is bounded. 
Step 2. We claim that $\left\|x_{n+1}-x_{n}\right\| \rightarrow 0$ and $\left\|y_{n+1}-y_{n}\right\| \rightarrow 0$ as $n \rightarrow \infty$. Indeed, from (9) we have

$$
\begin{aligned}
& \left\|x_{n+1}-x_{n}\right\| \\
& \leq\left\|\alpha_{n} f\left(x_{n}\right)+\left(I-\alpha_{n} \theta F\right) T^{n} y_{n}-\alpha_{n-1} f\left(x_{n-1}\right)-\left(I-\alpha_{n-1} \theta F\right) T^{n-1} y_{n-1}\right\| \\
& \leq \alpha_{n} \gamma\left\|x_{n}-x_{n-1}\right\|+\left|\alpha_{n}-\alpha_{n-1}\right|\left\|f\left(x_{n-1}\right)-\theta F T^{n} y_{n-1}\right\|+\left(1-\alpha_{n} \bar{\gamma} \theta\right)\left(\left\|y_{n}-y_{n-1}\right\|+c_{n}\right) \\
& \quad+\left(1-\alpha_{n-1} \bar{\gamma} \theta\right)\left\|T^{n} y_{n-1}-T^{n-1} y_{n-1}\right\| \\
& \leq \alpha_{n} \gamma\left\|x_{n}-x_{n-1}\right\|+\left|\alpha_{n}-\alpha_{n-1}\right| M_{1}+\left(1-\alpha_{n} \bar{\gamma} \theta\right)\left\|u_{n}-u_{n-1}\right\| \\
& \quad+\left\|T^{n} y_{n-1}-T^{n-1} y_{n-1}\right\|+c_{n},
\end{aligned}
$$

where

$$
M_{1}=\sup _{n \geq 1}\left\|\theta F T^{n} y_{n-1}-f\left(x_{n-1}\right)\right\| .
$$

Also, simple calculations show that

$$
\begin{aligned}
& \left\|u_{n}-u_{n-1}\right\|^{2} \\
& \leq \beta_{n}\left\|x_{n}-x_{n-1}\right\|\left\|u_{n}-u_{n-1}\right\|+\left(1-\beta_{n}\right)\left[\left\|S_{n} u_{n}-S_{n-1} u_{n}\right\|\left\|u_{n}-u_{n-1}\right\|\right. \\
& \left.\quad+\left\|u_{n}-u_{n-1}\right\|^{2}\right]+\left|\beta_{n}-\beta_{n-1}\right|\left\|x_{n-1}-S_{n-1} u_{n-1}\right\|\left\|u_{n}-u_{n-1}\right\| .
\end{aligned}
$$

So, it follows from (13) that

$$
\begin{aligned}
\left\|u_{n}-u_{n-1}\right\| \leq & \beta_{n}\left\|x_{n}-x_{n-1}\right\|+\left(1-\beta_{n}\right)\left[\left\|S_{n} u_{n}-S_{n-1} u_{n}\right\|\right. \\
& \left.+\left\|u_{n}-u_{n-1}\right\|\right]+\left|\beta_{n}-\beta_{n-1}\right|\left\|x_{n-1}-S_{n-1} u_{n-1}\right\|
\end{aligned}
$$

which immediately leads to

$$
\begin{aligned}
\left\|u_{n}-u_{n-1}\right\|-\left\|x_{n}-x_{n-1}\right\| & \leq \frac{1-\beta_{n}}{\beta_{n}}\left\|S_{n} u_{n}-S_{n-1} u_{n}\right\|+\left|\beta_{n}-\beta_{n-1}\right| \frac{\left\|x_{n-1}-S_{n-1} u_{n-1}\right\|}{\beta_{n}} \\
& \leq \frac{1}{a}\left\|S_{n} u_{n}-S_{n-1} u_{n}\right\|+\left|\beta_{n}-\beta_{n-1}\right| \frac{\left\|x_{n-1}-S_{n-1} u_{n-1}\right\|}{a} .
\end{aligned}
$$

Putting $D=\left\{u_{n}: n \geq 0\right\}$, we know that $D$ is a bounded subset of $C$. Then by the assumption we get $\sum_{n=1}^{\infty} \sup _{x \in D}\left\|S_{n} x-S_{n-1} x\right\|<\infty$. Noticing that

$$
\sup _{x \in D}\left\|S_{n} x-S_{n-1} x\right\| \geq\left\|S_{n} u_{n}-S_{n-1} u_{n}\right\|, \quad \forall n \geq 1,
$$

we have

$$
\sum_{n=1}^{\infty}\left\|S_{n} u_{n}-S_{n-1} u_{n}\right\|<\infty .
$$

Therefore, from (12) and (14) we deduce that

$$
\begin{aligned}
\left\|x_{n+1}-x_{n}\right\| \leq & \alpha_{n} \gamma\left\|x_{n}-x_{n-1}\right\|+\left|\alpha_{n}-\alpha_{n-1}\right| M_{1}+\left(1-\alpha_{n} \bar{\gamma} \theta\right)\left\{\left\|x_{n}-x_{n-1}\right\|\right. \\
& \left.+\frac{1}{a}\left\|S_{n} u_{n}-S_{n-1} u_{n}\right\|+\left|\beta_{n}-\beta_{n-1}\right| \frac{\left\|x_{n-1}-S_{n-1} u_{n-1}\right\|}{a}\right\} \\
& +c_{n}+\left\|T^{n} y_{n-1}-T^{n-1} y_{n-1}\right\| \\
\leq & \left|\alpha_{n}-\alpha_{n-1}\right| M_{1}+\frac{1}{a}\left\|S_{n} u_{n}-S_{n-1} u_{n}\right\|+\left[1-\alpha_{n}(\bar{\gamma} \theta-\gamma)\right]\left\|x_{n}-x_{n-1}\right\| \\
& +\left|\beta_{n}-\beta_{n-1}\right| \frac{\left\|x_{n-1}-S_{n-1} u_{n-1}\right\|}{a}+c_{n}+\left\|T^{n} y_{n-1}-T^{n-1} y_{n-1}\right\| \\
\leq & M_{2}\left(\left|\alpha_{n}-\alpha_{n-1}\right|+\left|\beta_{n}-\beta_{n-1}\right|+\left[1-\alpha_{n}(\bar{\gamma} \theta-\gamma)\right]\left\|x_{n}-x_{n-1}\right\|\right. \\
& \left.+\left\|S_{n} u_{n}-S_{n-1} u_{n}\right\|+c_{n}+\left\|T^{n} y_{n-1}-T^{n-1} y_{n-1}\right\|\right),
\end{aligned}
$$


where

$$
M_{2}=\sup _{n \geq 1}\left\{\frac{1}{a}+\frac{\left\|x_{n-1}-S_{n-1} u_{n-1}\right\|}{a}+M_{1}+1\right\} .
$$

From (15), the assumption $\sum_{n=0}^{\infty} c_{n}<\infty$ and conditions (i) and (ii), it can be readily seen that $\sum_{n=0}^{\infty} \alpha_{n}(\bar{\gamma} \theta-\gamma)=\infty$ and

$$
\sum_{n=1}^{\infty} M_{2}\left(\left\|S_{n} u_{n}-S_{n-1} u_{n}\right\|+c_{n}+\left|\alpha_{n}-\alpha_{n-1}\right|+\left|\beta_{n}-\beta_{n-1}\right|+\left\|T^{n} y_{n-1}-T^{n-1} y_{n-1}\right\|\right)<\infty .
$$

So, it follows from Lemma 7 and (16) that

$$
\lim _{n \rightarrow \infty}\left\|x_{n+1}-x_{n}\right\|=0
$$

Again from (9) and (14) we conclude that

$$
\begin{aligned}
& a\left\|y_{n}-y_{n-1}\right\|=a\left\|G u_{n}-G u_{n-1}\right\| \leq a\left\|u_{n}-u_{n-1}\right\| \\
& \leq a\left\|x_{n}-x_{n-1}\right\|+\left\|S_{n} u_{n}-S_{n-1} u_{n}\right\|+\left|\beta_{n}-\beta_{n-1}\right|\left\|x_{n-1}-S_{n-1} u_{n-1}\right\| \rightarrow 0 \quad(n \rightarrow \infty) .
\end{aligned}
$$

That is,

$$
\lim _{n \rightarrow \infty}\left\|y_{n+1}-y_{n}\right\|=0 \text {. }
$$

Step 3. We claim that $\left\|x_{n}-G x_{n}\right\| \rightarrow 0$ as $n \rightarrow \infty$. Indeed, note that $q=\Pi_{C}\left(p-\mu_{2} B_{2} p\right)$, $z_{n}=\Pi_{C}\left(u_{n}-\mu_{2} B_{2} u_{n}\right)$ and $y_{n}=\Pi_{C}\left(z_{n}-\mu_{1} B_{1} z_{n}\right)$. Then $y_{n}=G u_{n}$. By Lemma 4 we have

$$
\begin{aligned}
\left\|z_{n}-q\right\|^{2} & \leq\left\|u_{n}-p-\mu_{2}\left(B_{2} u_{n}-B_{2} p\right)\right\|^{2} \\
& \leq 2 \mu_{2}\left(\kappa^{2} \mu_{2}-\beta\right)\left\|B_{2} u_{n}-B_{2} p\right\|^{2}+\left\|u_{n}-p\right\|^{2}
\end{aligned}
$$

and

$$
\begin{aligned}
\left\|y_{n}-p\right\|^{2} & \leq\left\|z_{n}-q-\mu_{1}\left(B_{1} z_{n}-B_{1} q\right)\right\|^{2} \\
& \leq 2 \mu_{1}\left(\kappa^{2} \mu_{1}-\alpha\right)\left\|B_{1} z_{n}-B_{1} q\right\|^{2}+\left\|z_{n}-q\right\|^{2} .
\end{aligned}
$$

Substituting (19) for (20), we obtain

$$
\left\|y_{n}-p\right\|^{2}+2 \mu_{2}\left(\beta-\kappa^{2} \mu_{2}\right)\left\|B_{2} u_{n}-B_{2} p\right\|^{2}+2 \mu_{1}\left(\alpha-\kappa^{2} \mu_{1}\right)\left\|B_{1} z_{n}-B_{1} q\right\|^{2} \leq\left\|u_{n}-p\right\|^{2} .
$$

Let $v_{n}:=\left(T^{n} y_{n}-\alpha_{n} \theta F T^{n} y_{n}\right)+\alpha_{n} f\left(x_{n}\right)$. Then, from (7) and Lemma 2 we obtain

$$
\begin{aligned}
\left\|x_{n+1}-p\right\|^{2} & \leq\left\|v_{n}-p\right\|^{2} \\
& \leq 2 \alpha_{n}\left\langle f\left(x_{n}\right)-\theta F T^{n} y_{n}, j\left(v_{n}-p\right)\right\rangle+\left\|T^{n} y_{n}-p\right\|^{2} \\
& \leq 2 \alpha_{n}\left\|f\left(x_{n}\right)-\theta F T^{n} y_{n}\right\|\left\|v_{n}-p\right\|+\left(\left\|y_{n}-p\right\|+c_{n}\right)^{2} \\
& \leq\left\|y_{n}-p\right\|^{2}+c_{n} M_{2}+\alpha_{n} M_{3},
\end{aligned}
$$

where $M_{2}=\sup _{n \geq 0}\left\{2\left\|y_{n}-p\right\|+c_{n}\right\}$ and

$$
M_{3}=\sup _{n \geq 0}\left\{2\left\|f\left(x_{n}\right)-\theta F T^{n} y_{n}\right\|\left\|v_{n}-p\right\|\right\} .
$$

Substituting (21) to (22), we deduce from (10) that

$$
\begin{aligned}
& \left\|x_{n+1}-p\right\|^{2}+2 \mu_{2}\left(\beta-\kappa^{2} \mu_{2}\right)\left\|B_{2} u_{n}-B_{2} p\right\|^{2}+2 \mu_{1}\left(\alpha-\kappa^{2} \mu_{1}\right)\left\|B_{1} z_{n}-B_{1} q\right\|^{2} \\
& \leq\left\|x_{n}-p\right\|^{2}+c_{n} M_{2}+\alpha_{n} M_{3},
\end{aligned}
$$


which immediately yields

$$
\begin{aligned}
& 2 \mu_{2}\left(\beta-\kappa^{2} \mu_{2}\right)\left\|B_{2} u_{n}-B_{2} p\right\|^{2}+2 \mu_{1}\left(\alpha-\kappa^{2} \mu_{1}\right)\left\|B_{1} z_{n}-B_{1} q\right\|^{2} \\
& \leq \alpha_{n} M_{3}-\left\|x_{n+1}-p\right\|^{2}+c_{n} M_{2}+\left\|x_{n}-p\right\|^{2} \\
& \leq c_{n} M_{2}+\alpha_{n} M_{3}+\left(\left\|x_{n}-p\right\|+\left\|x_{n+1}-p\right\|\right)\left\|x_{n}-x_{n+1}\right\| .
\end{aligned}
$$

Since $\mu_{1} \kappa^{2} \in(0, \alpha), \mu_{2} \kappa^{2} \in(0, \beta), \lim _{n \rightarrow \infty} c_{n}=0$ and $\lim _{n \rightarrow \infty} \alpha_{n}=0$, we obtain from (17) that

$$
\lim _{n \rightarrow \infty}\left\|B_{2} u_{n}-B_{2} p\right\|=0 \quad \text { and } \quad \lim _{n \rightarrow \infty}\left\|B_{1} z_{n}-B_{1} q\right\|=0 .
$$

On the other hand, from Proposition 3 and Lemma 5 we have

$$
\begin{aligned}
2\left\|z_{n}-q\right\|^{2} & \leq 2\left\langle u_{n}-\mu_{2} B_{2} u_{n}-\left(p-\mu_{2} B_{2} p\right), j\left(z_{n}-q\right)\right\rangle \\
& \leq\left[\left\|u_{n}-p\right\|^{2}+\left\|z_{n}-q\right\|^{2}-g_{1}\left(\left\|u_{n}-z_{n}-(p-q)\right\|\right)\right]+2 \mu_{2}\left\|B_{2} p-B_{2} u_{n}\right\|\left\|z_{n}-q\right\|,
\end{aligned}
$$

which implies that

$$
\left\|z_{n}-q\right\|^{2}+g_{1}\left(\left\|u_{n}-z_{n}-(p-q)\right\|\right) \leq\left\|u_{n}-p\right\|^{2}+2 \mu_{2}\left\|B_{2} p-B_{2} u_{n}\right\|\left\|z_{n}-q\right\| .
$$

In the same way, we derive

$$
\begin{aligned}
2\left\|y_{n}-p\right\|^{2} & \leq 2\left\langle z_{n}-\mu_{1} B_{1} z_{n}-\left(q-\mu_{1} B_{1} q\right), j\left(y_{n}-p\right)\right\rangle \\
& \leq\left[\left\|z_{n}-q\right\|^{2}+\left\|y_{n}-p\right\|^{2}-g_{2}\left(\left\|z_{n}-y_{n}+(p-q)\right\|\right)\right]+2 \mu_{1}\left\|B_{1} q-B_{1} z_{n}\right\|\left\|y_{n}-p\right\|,
\end{aligned}
$$

which implies that

$$
\left\|y_{n}-p\right\|^{2}+g_{2}\left(\left\|z_{n}-y_{n}+(p-q)\right\|\right) \leq\left\|z_{n}-q\right\|^{2}+2 \mu_{1}\left\|B_{1} q-B_{1} z_{n}\right\|\left\|y_{n}-p\right\| .
$$

Substituting (24) for (25), we deduce from (10) that

$$
\begin{aligned}
\left\|y_{n}-p\right\|^{2} \leq & \left\|u_{n}-p\right\|^{2}-g_{1}\left(\left\|u_{n}-z_{n}-(p-q)\right\|\right)-g_{2}\left(\left\|z_{n}-y_{n}+(p-q)\right\|\right) \\
& +2 \mu_{2}\left\|B_{2} p-B_{2} u_{n}\right\|\left\|z_{n}-q\right\|+2 \mu_{1}\left\|B_{1} q-B_{1} z_{n}\right\|\left\|y_{n}-p\right\| \\
\leq & \left\|x_{n}-p\right\|^{2}-g_{1}\left(\left\|u_{n}-z_{n}-(p-q)\right\|\right)-g_{2}\left(\left\|z_{n}-y_{n}+(p-q)\right\|\right) \\
& +2 \mu_{1}\left\|B_{1} q-B_{1} z_{n}\right\|\left\|y_{n}-p\right\|+2 \mu_{2}\left\|B_{2} p-B_{2} u_{n}\right\|\left\|z_{n}-q\right\| .
\end{aligned}
$$

Substituting (26) for (22), we have

$$
\begin{aligned}
& \left\|x_{n+1}-p\right\|^{2}+g_{1}\left(\left\|u_{n}-z_{n}-(p-q)\right\|\right)+g_{2}\left(\left\|z_{n}-y_{n}+(p-q)\right\|\right) \\
& \leq\left\|x_{n}-p\right\|^{2}+2 \mu_{2}\left\|B_{2} p-B_{2} u_{n}\right\|\left\|z_{n}-q\right\|+2 \mu_{1}\left\|B_{1} q-B_{1} z_{n}\right\|\left\|y_{n}-p\right\|+c_{n} M_{2}+\alpha_{n} M_{3},
\end{aligned}
$$

which hence yields

$$
\begin{aligned}
& g_{1}\left(\left\|u_{n}-z_{n}-(p-q)\right\|\right)+g_{2}\left(\left\|z_{n}-y_{n}+(p-q)\right\|\right) \\
& \leq\left(\left\|x_{n}-p\right\|+\left\|x_{n+1}-p\right\|\right)\left\|x_{n}-x_{n+1}\right\|+2 \mu_{2}\left\|B_{2} p-B_{2} u_{n}\right\|\left\|z_{n}-q\right\| \\
& \quad+2 \mu_{1}\left\|B_{1} q-B_{1} z_{n}\right\|\left\|y_{n}-p\right\|+\alpha_{n} M_{3}+c_{n} M_{2} .
\end{aligned}
$$

Since $\lim _{n \rightarrow \infty} c_{n}=0$ and $\lim _{n \rightarrow \infty} \alpha_{n}=0$, we conclude from (17) and (23) that

$$
\lim _{n \rightarrow \infty} g_{1}\left(\left\|u_{n}-z_{n}-(p-q)\right\|\right)=0 \text { and } \lim _{n \rightarrow \infty} g_{2}\left(\left\|z_{n}-y_{n}+(p-q)\right\|\right)=0 .
$$


Using the properties of $g_{1}$ and $g_{2}$, we obtain

$$
\lim _{n \rightarrow \infty}\left\|u_{n}-z_{n}-(p-q)\right\|=0 \text { and } \lim _{n \rightarrow \infty}\left\|z_{n}-y_{n}+(p-q)\right\|=0 .
$$

It follows that

$$
\left\|u_{n}-y_{n}\right\| \leq\left\|u_{n}-z_{n}-(p-q)\right\|+\left\|z_{n}-y_{n}+(p-q)\right\| \rightarrow 0 \quad(n \rightarrow \infty) .
$$

That is,

$$
\lim _{n \rightarrow \infty}\left\|u_{n}-G u_{n}\right\|=\lim _{n \rightarrow \infty}\left\|u_{n}-y_{n}\right\|=0 .
$$

Also, according to (8) we have

$$
\left\|u_{n}-p\right\|^{2} \leq \beta_{n}\left\langle x_{n}-p, j\left(u_{n}-p\right)\right\rangle+\left(1-\beta_{n}\right)\left\|u_{n}-p\right\|^{2},
$$

which together with Lemma 5, yields

$$
\begin{aligned}
2\left\|u_{n}-p\right\|^{2} & \leq 2\left\langle x_{n}-p, j\left(u_{n}-p\right)\right\rangle \\
& \leq\left[\left\|x_{n}-p\right\|^{2}+\left\|u_{n}-p\right\|^{2}-g\left(\left\|x_{n}-u_{n}\right\|\right)\right] .
\end{aligned}
$$

This immediately implies that

$$
\left\|u_{n}-p\right\|^{2}+g\left(\left\|x_{n}-u_{n}\right\|\right) \leq\left\|x_{n}-p\right\|^{2},
$$

which together with (22), yields

$$
\begin{aligned}
\left\|x_{n+1}-p\right\|^{2} & \leq\left\|u_{n}-p\right\|^{2}+c_{n} M_{2}+\alpha_{n} M_{3} \\
& \leq\left\|x_{n}-p\right\|^{2}-g\left(\left\|x_{n}-u_{n}\right\|\right)+c_{n} M_{2}+\alpha_{n} M_{3} .
\end{aligned}
$$

Hence we have

$$
\begin{aligned}
g\left(\left\|u_{n}-x_{n}\right\|\right) & \leq\left\|x_{n}-p\right\|^{2}+c_{n} M_{2}-\left\|x_{n+1}-p\right\|^{2}+\alpha_{n} M_{3} \\
& \leq\left(\left\|x_{n}-p\right\|+\left\|x_{n+1}-p\right\|\right)\left\|x_{n}-x_{n+1}\right\|+\alpha_{n} M_{3}+c_{n} M_{2} .
\end{aligned}
$$

Since $\lim _{n \rightarrow \infty} c_{n}=0$ and $\lim _{n \rightarrow \infty} \alpha_{n}=0$, we obtain from (17) that $\lim _{n \rightarrow \infty} g\left(\left\|x_{n}-u_{n}\right\|\right)=0$. Using the properties of $g$, we have

$$
\lim _{n \rightarrow \infty}\left\|x_{n}-u_{n}\right\|=0
$$

Also, observe that

$$
\left\|x_{n}-u_{n}\right\|+\left\|u_{n}-G u_{n}\right\| \geq\left\|x_{n}-y_{n}\right\|,
$$

and

$$
\left\|x_{n}-y_{n}\right\|+\left\|u_{n}-x_{n}\right\| \geq\left\|x_{n}-y_{n}\right\|+\left\|G u_{n}-G x_{n}\right\| \geq\left\|x_{n}-G x_{n}\right\| .
$$

Then from (28) and (29) it follows that

$$
\lim _{n \rightarrow \infty}\left\|x_{n}-y_{n}\right\|=0 \quad \text { and } \quad \lim _{n \rightarrow \infty}\left\|x_{n}-G x_{n}\right\|=0 .
$$

Step 4. We claim that $\left\|T x_{n}-x_{n}\right\| \rightarrow 0$ and $\left\|S_{n} x_{n}-x_{n}\right\| \rightarrow 0$ as $n \rightarrow \infty$. Indeed, combining (8) with (29), we obtain that

$$
\left\|S_{n} u_{n}-u_{n}\right\|=\frac{\beta_{n}}{1-\beta_{n}}\left\|x_{n}-u_{n}\right\| \leq \frac{b}{1-b}\left\|x_{n}-u_{n}\right\| \rightarrow 0 \quad(n \rightarrow \infty) .
$$


That is,

$$
\lim _{n \rightarrow \infty}\left\|S_{n} u_{n}-u_{n}\right\|=0 .
$$

Since $\left\{S_{n}\right\}_{n=0}^{\infty}$ is $\ell$-uniformly Lipschitzian on $C$, we deduce from (29) and (31) that

$$
\begin{aligned}
\left\|S_{n} x_{n}-x_{n}\right\| & \leq\left\|S_{n} x_{n}-S_{n} u_{n}\right\|+\left\|S_{n} u_{n}-u_{n}\right\|+\left\|u_{n}-x_{n}\right\| \\
& \leq(\ell+1)\left\|x_{n}-u_{n}\right\|+\left\|S_{n} u_{n}-u_{n}\right\| \rightarrow 0 \quad(n \rightarrow \infty) .
\end{aligned}
$$

That is,

$$
\lim _{n \rightarrow \infty}\left\|x_{n}-S_{n} x_{n}\right\|=0
$$

We note that

$$
\begin{aligned}
\left\|x_{n}-T^{n} y_{n}\right\| & \leq\left\|x_{n}-x_{n+1}\right\|+\left\|x_{n+1}-T^{n} y_{n}\right\| \\
& =\leq\left\|x_{n}-x_{n+1}\right\|+\alpha_{n}\left\|f\left(x_{n}\right)-\theta F T^{n} y_{n}\right\| .
\end{aligned}
$$

Then we have

$$
\begin{aligned}
\left\|y_{n}-T^{n} y_{n}\right\| & \leq\left\|y_{n}-x_{n}\right\|+\left\|x_{n}-T^{n} y_{n}\right\| \\
& \leq\left\|y_{n}-x_{n}\right\|+\left\|x_{n}-x_{n+1}\right\|+\alpha_{n}\left\|f\left(x_{n}\right)-\theta F T^{n} y_{n}\right\| .
\end{aligned}
$$

Consequently, from (17), (30) and $\lim _{n \rightarrow \infty} \alpha_{n}=0$, it follows that

$$
\lim _{n \rightarrow \infty}\left\|y_{n}-T^{n} y_{n}\right\|=0
$$

We also note that

$$
\left\|y_{n}-T y_{n}\right\| \leq\left\|y_{n}-T^{n} y_{n}\right\|+\left\|T^{n} y_{n}-T^{n+1} y_{n}\right\|+\left\|T^{n+1} y_{n}-T y_{n}\right\| .
$$

By the assumption $\sum_{n=0}^{\infty}\left\|T^{n+1} y_{n}-T^{n} y_{n}\right\|<\infty$, (33) and the condition that $T: C \rightarrow C$ is uniformly continuous, we get

$$
\lim _{n \rightarrow \infty}\left\|y_{n}-T y_{n}\right\|=0
$$

In addition, noticing that

$$
\left\|x_{n}-T x_{n}\right\| \leq\left\|x_{n}-y_{n}\right\|+\left\|y_{n}-T y_{n}\right\|+\left\|T y_{n}-T x_{n}\right\|,
$$

we deduce from (30), (34) and the uniform continuity of $T$ that

$$
\lim _{n \rightarrow \infty}\left\|x_{n}-T x_{n}\right\|=0 .
$$

Step 5. We claim that $\left\|x_{n}-\bar{S} x_{n}\right\| \rightarrow 0$ as $n \rightarrow \infty$ where $\bar{S}:=(2 I-S)^{-1}$. Indeed, first, let us show that $S: C \rightarrow C$ is pseudocontractive and $\ell$-Lipschitzian such that $\lim _{n \rightarrow \infty}\left\|S x_{n}-x_{n}\right\|=0$ where $S x=$ $\lim _{n \rightarrow \infty} S_{n} x \forall x \in C$. Observe that for all $x, y \in C, \lim _{n \rightarrow \infty}\left\|S_{n} x-S x\right\|=0$ and $\lim _{n \rightarrow \infty}\left\|S_{n} y-S y\right\|=0$. Since $S_{n}$ is pseudocontractive operator, we get

$$
\langle S x-S y, j(x-y)\rangle \leq\|x-y\|^{2} .
$$

This means that $S$ is pseudocontractive. Noting that $\left\{S_{n}\right\}_{n=0}^{\infty}$ is $\ell$-uniformly Lipschitzian on $C$, we have

$$
\|S x-S y\|=\lim _{n \rightarrow \infty}\left\|S_{n} x-S_{n} y\right\| \leq \ell\|x-y\|, \quad \forall x, y \in C .
$$


This means that $S$ is $\ell$-Lipschitzian. Taking into account the boundedness of $\left\{x_{n}\right\}$ and putting $D=\overline{\operatorname{conv}}\left\{x_{n}: n \geq 0\right\}$ (the closed convex hull of the set $\left\{x_{n}: n \geq 0\right\}$ ), by the assumption we have $\sum_{n=1}^{\infty} \sup _{x \in D}\left\|S_{n-1} x-S_{n} x\right\|<\infty$. Hence, by Proposition 1 we get

$$
\lim _{n \rightarrow \infty} \sup _{x \in D}\left\|S_{n} x-S x\right\|=0
$$

which immediately yields

$$
\lim _{n \rightarrow \infty}\left\|S_{n} x_{n}-S x_{n}\right\|=0 .
$$

Thus, combining (32) with (36) we have

$$
\left\|x_{n}-S x_{n}\right\| \leq\left\|x_{n}-S_{n} x_{n}\right\|+\left\|S_{n} x_{n}-S x_{n}\right\| \rightarrow 0 \quad(n \rightarrow \infty) .
$$

That is,

$$
\lim _{n \rightarrow \infty}\left\|x_{n}-S x_{n}\right\|=0 .
$$

Now, let us show that if we define $\bar{S}:=(2 I-S)^{-1}$, then $\bar{S}: C \rightarrow C$ is nonexpansive, $\operatorname{Fix}(\bar{S})=\operatorname{Fix}(S)=\bigcap_{n=0}^{\infty} \operatorname{Fix}\left(S_{n}\right)$ and $\lim _{n \rightarrow \infty}\left\|x_{n}-\bar{S} x_{n}\right\|=0$. Indeed, put $\bar{S}:=(2 I-S)^{-1}$, where $I$ is the identity mapping on $E$. Then it is known that $\bar{S}$ is nonexpansive and the fixed-point set $\operatorname{Fix}(S)=\operatorname{Fix}(\bar{S})=\bigcap_{n=0}^{\infty} \operatorname{Fix}\left(S_{n}\right)$. From (37) it follows that

$$
\begin{aligned}
\left\|x_{n}-\bar{S} x_{n}\right\| & =\left\|\overline{S S}^{-1} x_{n}-\bar{S} x_{n}\right\| \\
& \leq\left\|\bar{S}^{-1} x_{n}-x_{n}\right\| \\
& =\left\|(2 I-S) x_{n}-x_{n}\right\|=\left\|x_{n}-S x_{n}\right\| \rightarrow 0 \quad(n \rightarrow \infty) .
\end{aligned}
$$

That is,

$$
\lim _{n \rightarrow \infty}\left\|x_{n}-\bar{S} x_{n}\right\|=0
$$

Step 6. We claim that

$$
\limsup _{n \rightarrow \infty}\left\langle f\left(x^{*}\right)-\theta F\left(x^{*}\right), j\left(x_{n}-x^{*}\right)\right\rangle \leq 0,
$$

where $x^{*}=\Pi_{\Omega}(f+I-\theta F)\left(x^{*}\right)$. Indeed, there exists a subsequence $\left\{x_{n_{i}}\right\}$ of $\left\{x_{n}\right\}$ such that

$$
\limsup _{n \rightarrow \infty}\left\langle f\left(x^{*}\right)-\theta F\left(x^{*}\right), j\left(x_{n}-x^{*}\right)\right\rangle=\lim _{i \rightarrow \infty}\left\langle f\left(x^{*}\right)-\theta F\left(x^{*}\right), j\left(x_{n_{i}}-x^{*}\right)\right\rangle .
$$

Now we show that $\Pi_{\Omega}(f+I-\theta F)$ is a contraction mapping. Since $F$ is bounded linear strongly positive, for all $x, y \in C$, we have

$$
\begin{aligned}
& \left\|\Pi_{\Omega}(f+I-\theta F)(x)-\Pi_{\Omega}(f+I-\theta F)(y)\right\| \\
& \leq\|f(x)-f(y)\|+\|(I-\theta F)(x)-(I-\theta F)(y)\| \\
& \leq[1+(\gamma-\bar{\gamma} \theta)]\|x-y\|,
\end{aligned}
$$

which implies that $\Pi_{\Omega}(f+I-\theta F)$ is a contraction mapping. Banach's contraction mapping principle guarantees that $\Pi_{\Omega}(f+I-\theta F)$ has a unique fixed point. Say $x^{*} \in C$, that is, $x^{*}=\Pi_{\Omega}(f+I-\theta F)\left(x^{*}\right)$. Since $\left\{x_{n}\right\}$ is a bounded sequence in $C$, we may assume that $x_{n_{i}} \rightarrow \bar{x} \in C$. Please note that $G$ and $\bar{S}$ are nonexpansive and that $T$ is asymptotically nonexpansive in the intermediate sense. Since $(I-G) x_{n} \rightarrow 0$ and $(I-\bar{S}) x_{n} \rightarrow 0$ (due to (30) and (37)), by Lemma 6 we have that $\bar{x} \in \operatorname{Fix}(G)=$ $\operatorname{GSVI}\left(C, B_{1}, B_{2}\right)$ and $\bar{x} \in \operatorname{Fix}(\bar{S})=\operatorname{Fix}(S)=\bigcap_{n=0}^{\infty} \operatorname{Fix}\left(S_{n}\right)$. From (35), we have that $\lim _{i \rightarrow \infty} \| x_{n_{i}}-$ $T x_{n_{i}} \|=0$ for the subsequence $\left\{x_{n_{i}}\right\}$ of $\left\{x_{n}\right\}$. It follows that $\bar{x} \in \operatorname{Fix}(T)$. Then, $\bar{x} \in \Omega=\bigcap_{n=0}^{\infty} \operatorname{Fix}\left(S_{n}\right) \cap$ 
$\operatorname{GSVI}\left(C, B_{1}, B_{2}\right) \cap \operatorname{Fix}(T)$. Since $E$ admits a weakly sequentially continuous duality mapping $j(\cdot)$ and $x_{n_{i}} \rightarrow \bar{x}$, we obtain

$$
\begin{aligned}
\limsup _{n \rightarrow \infty}\left\langle f\left(x^{*}\right)-\theta F\left(x^{*}\right), j\left(x_{n}-x^{*}\right)\right\rangle & =\lim _{i \rightarrow \infty}\left\langle f\left(x^{*}\right)-\theta F\left(x^{*}\right), j\left(x_{n_{i}}-x^{*}\right)\right\rangle \\
& =\left\langle f\left(x^{*}\right)-\theta F\left(x^{*}\right), j\left(\bar{x}-x^{*}\right)\right\rangle \leq 0,
\end{aligned}
$$

which implies that (39) holds. Noticing that $j(\cdot)$ is also norm-to-norm uniformly continuous on bounded subsets of $E$, we obtain from (17) that

$$
\limsup _{n \rightarrow \infty}\left\langle f\left(x^{*}\right)-\theta F\left(x^{*}\right), j\left(x_{n+1}-x^{*}\right)\right\rangle \leq 0 .
$$

Step 7. We claim that $x_{n} \rightarrow x^{*}$ as $n \rightarrow \infty$. Indeed, it follows from $x_{n+1}=\Pi_{C} v_{n}$ and Proposition 3 (iii) that

$$
\left\langle\Pi_{C} v_{n}-v_{n}, j\left(\Pi_{C} v_{n}-x^{*}\right)\right\rangle \leq 0,
$$

which leads to

$$
\left\langle x_{n+1}-v_{n}, j\left(x_{n+1}-x^{*}\right)\right\rangle \leq 0 .
$$

Then, from (11) we have

$$
\begin{aligned}
2 & \left\|x_{n+1}-x^{*}\right\|^{2} \\
\leq & 2\left\langle x^{*}-v_{n}, j\left(x^{*}-x_{n+1}\right)\right\rangle \\
\leq & 2 \alpha_{n} \gamma\left\|x_{n}-x^{*}\right\|\left\|x_{n+1}-x^{*}\right\|+2\left(1-\alpha_{n} \bar{\gamma} \theta\right)\left(\left\|y_{n}-x^{*}\right\|+c_{n}\right)\left\|x_{n+1}-x^{*}\right\| \\
& +2 \alpha_{n}\left\langle f\left(x^{*}\right)-\theta F\left(x^{*}\right), j\left(x_{n+1}-x^{*}\right)\right\rangle \\
\leq & 2\left[1-\alpha_{n}(\bar{\gamma} \theta-\gamma)\right]\left\|x_{n}-x^{*}\right\|\left\|x_{n+1}-x^{*}\right\|+2 c_{n} M_{4}+2 \alpha_{n}\left\langle f\left(x^{*}\right)-\theta F\left(x^{*}\right), j\left(x_{n+1}-x^{*}\right)\right\rangle \\
\leq & \left\|x_{n+1}-x^{*}\right\|^{2}+\left(1-\alpha_{n}(\bar{\gamma} \theta-\gamma)\right)\left\|x_{n}-x^{*}\right\|^{2}+2 c_{n} M_{4}+2 \alpha_{n}\left\langle f\left(x^{*}\right)-\theta F\left(x^{*}\right), j\left(x_{n+1}-x^{*}\right)\right\rangle,
\end{aligned}
$$

where $M_{4}=\sup _{n \geq 0}\left\|x_{n+1}-x^{*}\right\|$. This immediately implies that

$$
\left\|x_{n+1}-x^{*}\right\|^{2} \leq\left[1-\alpha_{n}(\bar{\gamma} \theta-\gamma)\right]\left\|x_{n}-x^{*}\right\|^{2}+\alpha_{n}(\bar{\gamma} \theta-\gamma) \frac{2\left\langle f\left(x^{*}\right)-\theta F\left(x^{*}\right), j\left(x_{n+1}-x^{*}\right)\right\rangle}{\bar{\gamma} \theta-\gamma}+2 c_{n} M_{4} .
$$

Applying Lemma 7 to (41), we infer that $x_{n} \rightarrow x^{*}$ as $n \rightarrow \infty$. The proof is completed.

It is remarkable that according to the proof of Theorem 1, we know that $\left\{y_{n}\right\}$ is bounded. We now give two examples to illustrate partial conditions of Theorem 1 to be satisfied.

Example 1. Let $T: C \rightarrow C$ be a contraction mapping with a constant $\beta \in(0,1)$. We take $S_{n}:=T^{n}$ and obtain

$$
\sup _{x \in D}\left\|S_{n} x-S_{n-1} x\right\|=\sup _{x \in D}\left\|T^{n} x-T^{n-1} x\right\| \leq \beta^{n-1} \cdot \sup _{x \in D}\|T x-x\|, \quad \forall n \geq 1,
$$

for any bounded subset $D$ of $C$. Therefore, it follows that

$$
+\infty>\sum_{n=1}^{\infty} \beta^{n-1} \cdot \sup _{x \in D}\|T x-x\| \geq \sum_{n=1}^{\infty} \sup _{x \in D}\left\|S_{n} x-S_{n-1} x\right\| .
$$

In particular, whenever $D$ is a bounded sequence $\left\{x_{n}\right\}_{n=0}^{\infty}$ in $C$, we have

$$
+\infty>\sum_{n=0}^{\infty} \sup _{x \in D}\left\|S_{n+1} x-S_{n} x\right\| \geq \sum_{n=0}^{\infty}\left\|S_{n+1} x_{n}-S_{n} x_{n}\right\|=\sum_{n=0}^{\infty}\left\|T^{n+1} x_{n}-T^{n} x_{n}\right\| .
$$


Since $T$ is a contraction mapping, Banach's Contraction Mapping Principle guarantees that $T$ has a unique fixed point. Say $p \in C$. We define $S x:=p$ for all $x \in C$. It is easy to see that $S x=\lim _{n \rightarrow \infty} S_{n} x$ for all $x \in C$ and $\operatorname{Fix}(S)=\bigcap_{n=0}^{\infty} \operatorname{Fix}\left(S_{n}\right)$.

Example 2. Let $E=\mathbf{R}$ and $C=[-1,1]$, and let $T: C \rightarrow C$ be an identity mapping, i.e., $T x=x$ for all $x \in C$. Moreover, we define

$$
S_{n} x:=\frac{\sin x+x}{2+n}, \quad \forall x \in C, \forall n \geq 0 .
$$

Then we obtain

$$
\begin{aligned}
& \left|S_{n} x-S_{n} y\right|=\left|\frac{\sin x+}{n+2}-\frac{\sin y+y}{n+2}\right| \\
& \leq \frac{|y-x|+|\sin y-\sin x|}{n+2} \leq \frac{2|x-y|}{n+2} \leq|x-y|, \quad \forall x, y \in C, \forall n \geq 0,
\end{aligned}
$$

and

$$
\begin{aligned}
\sup _{x \in C}\left|S_{n} x-S_{n-1} x\right| & =\sup _{x \in C}\left|\frac{x+\sin x}{n+2}-\frac{x+\sin x}{n+1}\right|=\sup _{x \in C}\left|\frac{x+\sin x}{(n+2)(n+1)}\right| \\
& \leq \frac{2}{(n+2)(n+1)} \leq \frac{2}{(n+1)^{2}}, \quad \forall n \geq 0 .
\end{aligned}
$$

Therefore, it follows that for any bounded subset $D$ of $C$,

$$
\sum_{n=1}^{\infty} \sup _{x \in D}\left|S_{n} x-S_{n-1} x\right|<\infty
$$

In addition, whenever $\left\{x_{n}\right\}_{n=0}^{\infty}$ is a bounded sequence in $C$, it is clear that

$$
\sum_{n=0}^{\infty}\left|T^{n} x_{n}-T^{n+1} x_{n}\right|<\infty
$$

Also, we define $S x:=0$ for all $x \in C$. Then, it is clear that $S x=\lim _{n \rightarrow \infty} S_{n} x \forall x \in C$, and $\operatorname{Fix}(S)=\bigcap_{n=0}^{\infty} \operatorname{Fix}\left(S_{n}\right)$.

\section{Applications}

Now, we give an application to solve CFPPs of asymptotically nonexpansive and pseudocontractive mappings, and variational inequality problems for strict pseudocontractive mappings in Banach spaces.

Let $C$ be a nonempty, closed, and convex subset of a real Banach space $E$. A mapping $T: C \rightarrow C$ is said to be $\lambda$-strictly pseudocontractive if for every $x, y \in C$ there exists $j(x-y) \in J(x-y)$ such that

$$
\langle T x-T y, j(x-y)\rangle+\lambda\|(I-T) x-(I-T) y\|^{2} \leq\|x-y\|^{2}, \quad \text { for some } \lambda \in(0,1) .
$$

A simple computation shows that (42) is equivalent to the following inequality:

$$
\langle(I-T) x-(I-T) y, j(x-y)\rangle \geq \lambda\|(I-T) x-(I-T) y\|^{2},
$$

for every $x, y \in C$ and for some $j(x-y) \in J(x-y)$. Therefore, $I-T$ is $\lambda$-inverse-strongly accretive.

By Theorem 1, we can obtain the following results easily.

Theorem 2. Let $C$ be a convex closed set in a 2-uniformly smooth and uniformly convex Banach space $E$ which admits a weakly sequentially continuous duality mapping. Let $\Pi_{C}$ be a sunny nonexpansive retraction from $E$ onto $C$. Let the mappings $B_{1}, B_{2}: C \rightarrow C$ be $\alpha$-strictly pseudocontractive and $\beta$-strictly pseudocontractive, respectively. Let $f: C \rightarrow C$ be a contraction mapping with coefficient $\gamma \in[0,1)$ and $F: E \rightarrow E$ be a strongly 
positive linear bounded operator with the coefficient $\bar{\gamma}$ such that $0<\gamma<\bar{\gamma} \theta$ and $0<\theta \leq\|F\|^{-1}$. Let $T: C \rightarrow C$ be uniformly continuous and asymptotically nonexpansive mapping in the intermediate sense, and $\left\{S_{n}\right\}_{n=0}^{\infty}$ be a countable family of $\ell$-uniformly Lipschitzian pseudocontractive self-mappings on $C$ such that $\Omega:=\bigcap_{n=0}^{\infty} \operatorname{Fix}\left(S_{n}\right) \cap \operatorname{GSVI}\left(C, I-B_{1}, I-B_{2}\right) \cap \operatorname{Fix}(T) \neq \varnothing$ where $\operatorname{GSVI}\left(C, I-B_{1}, I-B_{2}\right)$ is the fixed-point set of the mapping $G:=\left[\left(1-\mu_{1}\right) I+\mu_{1} B_{1}\right]\left[\left(1-\mu_{2}\right) I+\mu_{2} B_{2}\right]$ with $0<\mu_{1} \kappa^{2}<\min \{1, \alpha\}$ and $0<\mu_{2} \kappa^{2}<\min \{1, \beta\}$ for $\kappa$ the 2 -uniformly smooth constant of $E$. Assume that $\sum_{n=0}^{\infty} c_{n}<\infty$, where $c_{n}$ is defined by (2). For arbitrarily given $x_{0} \in C$, let $\left\{x_{n}\right\}$ be the sequence generated by

$$
\left\{\begin{array}{l}
y_{n}=\left(1-\mu_{1}\right) z_{n}+\mu_{1} B_{1} z_{n} \\
z_{n}=\left(1-\mu_{2}\right) u_{n}+\mu_{2} B_{2} u_{n} \\
u_{n}=\beta_{n}\left(x_{n}-S_{n} u_{n}\right)+S_{n} u_{n} \\
x_{n+1}=\Pi_{C}\left[\left(T^{n} y_{n}-\alpha_{n} \theta F T^{n} y_{n}\right)+\alpha_{n} f\left(x_{n}\right)\right], \quad \forall n \geq 0,
\end{array}\right.
$$

where $\left\{\alpha_{n}\right\}$ and $\left\{\beta_{n}\right\}$ are the sequences in $[0,1]$ satisfying the following conditions:

(i) $\sum_{n=0}^{\infty} \alpha_{n}=\infty, \sum_{n=1}^{\infty}\left|\alpha_{n}-\alpha_{n-1}\right|<\infty$ and $\lim _{n \rightarrow \infty} \alpha_{n}=0$;

(ii) $0<\liminf _{n \rightarrow \infty} \beta_{n} \leq \limsup _{n \rightarrow \infty} \beta_{n}<1$ and $\sum_{n=1}^{\infty}\left|\beta_{n}-\beta_{n-1}\right|<\infty$.

Assume that $\sum_{n=1}^{\infty} \sup _{x \in D}\left\|S_{n} x-S_{n-1} x\right\|<\infty$ for any bounded subset $D$ of $C$, and let $S$ be a mapping of $C$ into itself defined by $S x=\lim _{n \rightarrow \infty} S_{n} x$ for all $x \in C$, and suppose that $\operatorname{Fix}(S)=\bigcap_{n=0}^{\infty} \operatorname{Fix}\left(S_{n}\right)$. If $\sum_{n=0}^{\infty}\left\|T^{n+1} y_{n}-T^{n} y_{n}\right\|<\infty$, then $\left\{x_{n}\right\}$ converges strongly to $x^{*} \in \Omega$. In this case,

(a) $x^{*} \in \Omega$ solves the VI: $\left\langle f\left(x^{*}\right)-\theta F\left(x^{*}\right), j\left(x-x^{*}\right)\right\rangle \leq 0, \forall x \in \Omega$;

(b) $\left(x^{*}, y^{*}\right)$ is a solution of GSVI (1.3) for two inverse-strongly accretive mappings $I-B_{i}, i=1,2$, where $y^{*}=\left(1-\mu_{2}\right) x^{*}+\mu_{2} B_{2} x^{*}$.

Proof. Since the mappings $B_{1}, B_{2}: C \rightarrow C$ are $\alpha$-strictly pseudocontractive and $\beta$-strictly pseudocontractive, respectively, it can be seen readily that $I-B_{1}, I-B_{2}: C \rightarrow E$ are $\alpha$-inverse-strongly accretive and $\beta$-inverse-strongly accretive, respectively. Please note that $0<\mu_{1} \kappa^{2}<\min \{1, \alpha\}$ and $0<\mu_{2} \kappa^{2}<\min \{1, \beta\}$ for $\kappa$ the 2 -uniformly smooth constant of $E$. Then, GSVI $\left(C, I-B_{1}, I-B_{2}\right)$ is the fixed-point set of the following mapping

$$
\begin{aligned}
G x: & =\Pi_{C}\left[\left(1-\mu_{1}\right) I+\mu_{1} B_{1}\right] \Pi_{C}\left[\left(1-\mu_{2}\right) x+\mu_{2} B_{2} x\right] \\
& =\Pi_{C}\left\{\left(1-\mu_{1}\right)\left[\left(1-\mu_{2}\right) x+\mu_{2} B_{2} x\right]+\mu_{1} B_{1}\left[\left(1-\mu_{2}\right) x+\mu_{2} B_{2} x\right]\right\} \\
& =\left[\left(1-\mu_{1}\right) I+\mu_{1} B_{1}\right]\left[\left(1-\mu_{2}\right) I+\mu_{2} B_{2}\right] x, \quad \forall x \in C .
\end{aligned}
$$

In this case, it is easy to see that the iterative scheme (7) reduces to (44). Therefore, by Theorem 1 we obtain the desired result.

Theorem 3. Let $C$ be a bounded, convex and closed set in a 2-uniformly smooth and uniformly convex Banach space $E$ which admits a weakly sequentially continuous duality mapping. Let $\Pi_{C}$ be a sunny nonexpansive retraction from $E$ onto $C$. Let the mappings $B_{1}, B_{2}: C \rightarrow C$ be $\alpha$-strictly pseudocontractive and $\beta$-strictly pseudocontractive, respectively. Let $f: C \rightarrow C$ be a contraction mapping with coefficient $\gamma \in[0,1)$ and $F: E \rightarrow$ E be a strongly positive linear bounded operator with the coefficient $\bar{\gamma}$ such that $0<\gamma<\bar{\gamma} \theta$ and $0<\theta \leq\|F\|^{-1}$. Let $T: C \rightarrow C$ be an asymptotically nonexpansive mapping with a sequence $\left\{\theta_{n}\right\} \subset[0, \infty)$ satisfying $\sum_{n=0}^{\infty} \theta_{n}<\infty$, and $\left\{S_{n}\right\}_{n=0}^{\infty}$ be a countable family of $\ell$-uniformly Lipschitzian pseudocontractive self-mappings on $C$ such that $\Omega:=\bigcap_{n=0}^{\infty} \operatorname{Fix}\left(S_{n}\right) \cap \operatorname{GSVI}\left(C, I-B_{1}, I-B_{2}\right) \cap \operatorname{Fix}(T) \neq \varnothing$ where $\operatorname{GSVI}\left(C, I-B_{1}, I-B_{2}\right)$ is the fixed-point set of the mapping $G:=\left[\left(1-\mu_{1}\right) I+\mu_{1} B_{1}\right]\left[\left(1-\mu_{2}\right) I+\mu_{2} B_{2}\right]$ with $0<\mu_{1} \kappa^{2}<\min \{1, \alpha\}$ 
and $0<\mu_{2} \kappa^{2}<\min \{1, \beta\}$ for $\kappa$ the 2-uniformly smooth constant of $E$. For arbitrarily given $x_{0} \in C$, let $\left\{x_{n}\right\}$ be the sequence generated by

$$
\left\{\begin{array}{l}
z_{n}=\left(1-\mu_{2}\right) u_{n}+\mu_{2} B_{2} u_{n} \\
u_{n}=\beta_{n}\left(x_{n}-S_{n} u_{n}\right)+S_{n} u_{n} \\
y_{n}=\left(1-\mu_{1}\right) z_{n}+\mu_{1} B_{1} z_{n} \\
x_{n+1}=\Pi_{C}\left[\alpha_{n} f\left(x_{n}\right)+\left(I-\alpha_{n} \theta F\right) T^{n} y_{n}\right], \quad \forall n \geq 0
\end{array}\right.
$$

where $\left\{\alpha_{n}\right\}$ and $\left\{\beta_{n}\right\}$ are the sequences in $[0,1]$ satisfying the following conditions:

(i) $\sum_{n=0}^{\infty} \alpha_{n}=\infty, \sum_{n=1}^{\infty}\left|\alpha_{n}-\alpha_{n-1}\right|<\infty$ and $\lim _{n \rightarrow \infty} \alpha_{n}=0$;

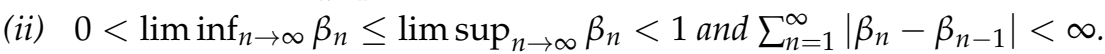

Assume that $\sum_{n=1}^{\infty} \sup _{x \in D}\left\|S_{n} x-S_{n-1} x\right\|<\infty$ for any bounded subset $D$ of $C$, and let $S$ be a mapping of $C$ into itself defined by $S x=\lim _{n \rightarrow \infty} S_{n} x$ for all $x \in C$, and suppose that $\operatorname{Fix}(S)=\bigcap_{n=0}^{\infty} \operatorname{Fix}\left(S_{n}\right)$. If $\sum_{n=0}^{\infty}\left\|T^{n+1} y_{n}-T^{n} y_{n}\right\|<\infty$, then $\left\{x_{n}\right\}$ converges strongly to $x^{*} \in \Omega$. In this case,

(a) $x^{*} \in \Omega$ solves the VI: $\left\langle f\left(x^{*}\right)-\theta F\left(x^{*}\right), j\left(x-x^{*}\right)\right\rangle \leq 0, \forall x \in \Omega$;

(b) $\left(x^{*}, y^{*}\right)$ is a solution of GSVI (1.3) for two inverse-strongly accretive mappings $I-B_{i}, i=1,2$, where $y^{*}=\left(1-\mu_{2}\right) x^{*}+\mu_{2} B_{2} x^{*}$.

Proof. Since set $C$ is a bounded set, we know that $\operatorname{diam}(C)=\sup _{x, y \in C}\|x-y\|<\infty$. We have that $T: C \rightarrow C$ is an asymptotically nonexpansive mapping with a sequence $\left\{\theta_{n}\right\} \subset[0, \infty)$ satisfying $\sum_{n=0}^{\infty} \theta_{n}<\infty$. Then, we deduce that for all $x, y \in C$,

$$
\left\|T^{n} x-T^{n} y\right\| \leq\left(1+\theta_{n}\right)\|x-y\|=\|x-y\|+\theta_{n}\|x-y\| \leq\|x-y\|+\theta_{n} \operatorname{diam}(C) .
$$

Hence, we get

$$
c_{n}:=\max \left\{0, \sup _{x, y \in C}\left(\left\|T^{n} x-T^{n} y\right\|-\|x-y\|\right)\right\} \leq \theta_{n} \operatorname{diam}(C),
$$

which immediately attains $\sum_{n=0}^{\infty} c_{n}<\infty$. Therefore, by Theorem 1 we derive the desired result.

\section{Conclusions}

In this work, we studied problem of solving a general system of monotone variational inequalities whose solutions are also the solutions of the CFPP of countably many nonlinear operators via a hybrid viscosity implicit iteration method. Strong convergence theorems were established in 2-uniformly smooth and uniformly convex Banach spaces. An application to CFPPs of asymptotically nonexpansive and pseudocontractive mappings, and variational inequality problems for strict pseudocontractive mappings was also given in Banach spaces. We also provided two examples to support the main results of this paper.

Author Contributions: These authors contributed equally to this work.

Funding: This research was funded by the Natural Science Foundation of Shandong Province of China (ZR2017LA001) and Youth Foundation of Linyi University (LYDX2016BS023). The first author was partially supported by the Innovation Program of Shanghai Municipal Education Commission (15ZZ068), Ph.D. Program Foundation of Ministry of Education of China (20123127110002) and Program for Outstanding Academic Leaders in Shanghai City (15XD1503100).

Conflicts of Interest: The authors declare no conflict of interest. 


\section{References}

1. Goebel, K.; Kirk, W.A. A fixed point theorem for asymptotically nonexpansive mappings. Proc. Am. Math. Soc. 1972, 35, 171-174. [CrossRef]

2. Bruck, R.E.; Kuczumow, T.; Reich, S. Convergence of iterates of asymptotically nonexpansive mappings in Banach spaces with the uniform Opial property. Colloq. Math. 1993, 65, 169-179. [CrossRef]

3. Kato, T. Nonlinear semigroups and evolution equations. J. Math. Soc. Jpn. 1967, 19, 508-520. [CrossRef]

4. Qin, X.; Cho, S.Y.; Wang, L. Strong convergence of an iterative algorithm involving nonlinear mappings of nonexpansive and accretive type. Optimization 2018, 67, 1377-1388. [CrossRef]

5. Qin, X.; Cho, S.Y. Convergence analysis of a monotone projection algorithm in reflexive Banach spaces. Acta Math. Sci. 2017, 37, 488-502. [CrossRef]

6. Zhao, X.; Ng, K.F.; Li, C.; Yao, J.C. Linear regularity and linear convergence of projection-based methods for solving convex feasibility problems. Appl. Math. Optim. 2018, 78, 613-641. [CrossRef]

7. Bin Dehaish, B.A. A regularization projection algorithm for various problems with nonlinear mappings in Hilbert spaces. J. Inequal. Appl. 2015, 2015, 51. [CrossRef]

8. Ceng, L.C. Hybrid viscosity approximation method for zeros of m-accretive operators in Banach spaces. Numer. Funct. Anal. Optim. 2012, 33, 142-165. [CrossRef]

9. Ceng, L.C. Hybrid viscosity extragradient method for systems of variational inequalities, fixed points of nonexpansive mappings, zero points of accretive operators in Banach spaces. Fixed Point Theory 2018, 19, 487-502. [CrossRef]

10. Takahashi, W.; Wen, C.F.; Yao, J.C. The shrinking projection method for a finite family of demimetric mappings with variational inequality problems in a Hilbert space. Fixed Point Theory 2018, 19, 407-419. [CrossRef]

11. Ansari, Q.H.; Babu, F.; Yao, J.C. Regularization of proximal point algorithms in Hadamard manifolds. J. Fixed Point Theory Appl. 2019, 21, 25. [CrossRef]

12. Chang, S.S.; Wen, C.F.; Yao, J.C. Common zero point for a finite family of inclusion problems of accretive mappings in Banach spaces. Optimization 2018, 67, 1183-1196. [CrossRef]

13. Bin Dehaish, B.A. Weak and strong convergence of algorithms for the sum of two accretive operators with applications. J. Nonlinear Convex Anal. 2015, 16, 1321-1336.

14. Ceng, L.C.; Wang, C.Y.; Yao, J.C. Strong convergence theorems by a relaxed extragradient method for a general system of variational inequalities. Math. Methods Oper. Res. 2008, 67, 375-390. [CrossRef]

15. Cho, S.Y. Weak convergence of a splitting algorithm in Hilbert spaces. J. Appl. Anal. Comput. 2017, 7, 427-438.

16. Cai, G.; Bu, S.Q. Convergence analysis for variational inequality problems and fixed point problems in 2-uniformly smooth and uniformly convex Banach spaces. Math. Comput. Model. 2012, 55, 538-546. [CrossRef]

17. Qin, X.; Yao, J.C. Weak convergence of a Mann-like algorithm for nonexpansive and accretive operators. J. Inequal. Appl. 2016, 2016, 232. [CrossRef]

18. Qin, X.; Yao, J.C. Projection splitting algorithms for nonself operators. J. Nonlinear Convex Anal. 2017, 18, 925-935.

19. Cholamjiak, W.; Suantai, S.; Suparatulatorn, R.; Kesornprom, S.; Cholamjiak, P. Viscosity approximation methods for fixed point problems in Hilbert spaces endowed with graphs. J. Appl. Numer. Optim. 2019, 1, $25-38$.

20. Nguyen, L.V.; Qin, X. Some results on strongly pseudomonotone quasi-variational inequalities. Set-Valued Var. Anal. 2019. [CrossRef]

21. Ceng, L.C. Convergence analysis of a Mann-like iterative algorithm in reflexive Banach spaces. Appl. Set-Valued Anal. Optim. 2019, 1, 1-18.

22. Qin, X.; Petrusel, A.; Yao, J.C. CQ iterative algorithms for fixed points of nonexpansive mappings and split feasibility problems in Hilbert spaces. J. Nonlinear Convex Anal. 2018, 19, 157-165.

23. Ball, K.; Carlen, E.A.; Lieb, E.H. Sharp uniform convexity and smoothness inequalities for trace norms. Invent. Math. 2994, 115, 463-482. [CrossRef]

24. Takahashi, Y.; Hashimoto, K.; Kato, M. On sharp uniform convexity, smoothness, and strong type, cotype inequalities. J. Nonlinear Convex Anal. 2002, 3, 267-281. 
25. Aoyama, K.; Kimura, Y.; Takahashi, W.; Toyoda, M. Approximation of common fixed points of a countable family of nonexpansive mappings in a Banach space. Nonlinear Anal. 2007, 67, 2350-2360. [CrossRef]

26. Deimling, K. Zeros of accretive operators. Manuscr. Math. 1974, 13, 365-374. [CrossRef]

27. Gossez, J.P.; Lami Dozo, E. Some geometric properties related to the fixed point theory for nonexpansive mappings. Pac. J. Math. 1972, 40, 565-573. [CrossRef]

28. Reich, S. On the asymptotical behavior of nonlinear semigroups and the range of accretive operators. J. Math. Anal. Appl. 1981, 79, 113-126. [CrossRef]

29. Reich, S. Weak convergence theorems for nonexpansive mappings in Banach spaces. J. Math. Anal. Appl. 1979, 67, 274-276. [CrossRef]

30. Qin, X.; Cho, S.Y.; Yao, J.C. Weak and strong convergence of splitting algorithms in Banach spaces. Optimization 2019, in press.

31. Reich, S. Product formulas, nonlinear semigroups, and accretive operators. J. Funct. Anal. 1980, 36, 147-168. [CrossRef]

32. Qin, X.; Cho, S.Y.; Wang, L. Iterative algorithms with errors for zero points of m-accretive operators. Fixed Point Theory Appl. 2013, 2013, 148. [CrossRef]

33. Oka, H. An ergodic theorem for asymptotically nonexpansive mappings in the intermediate sense. Proc. Am. Math. Soc. 1997, 125, 1693-1703. [CrossRef]

34. Kamimura, S.; Takahashi, W. Strong convergence of a proximal-type algorithm in a Banach space. SIAM J. Optim. 2002, 13, 938-945. [CrossRef]

35. Goebel, K. Kirk, W.A. Cambridge Studies in Advanced Mathematics. In Topics in Metric Fixed Point Theory; Cambridge University Press: Cambridge, UK, 1990; Volume 28.

36. Xue, Z.; Zhou, H.; Cho, Y.J. Iterative solutions of nonlinear equations for $\mathrm{m}$-accretive operators in Banach spaces. J. Nonlinear Convex Anal. 2000, 1, 313-320.

(C) 2019 by the authors. Licensee MDPI, Basel, Switzerland. This article is an open access article distributed under the terms and conditions of the Creative Commons Attribution (CC BY) license (http:/ / creativecommons.org/licenses/by/4.0/). 